\title{
Nurturing the Continuum of HIV Testing, Treatment and Prevention Matrix Cascade in Reducing HIV Transmission
}

\author{
Clarence S Yah ${ }^{1}$
}

\author{
OPEN ACCESS \\ Citation: Clarence S Yah. Nurturing the \\ Continuum of HIV Testing, Treatment \\ and Prevention Matrix Cascade in \\ Reducing HIV Transmission. Ethiop J \\ Health Sci.2017;27(6):621 \\ doi:http://dx.doi.org/10.4314/ejhs.v27i6.7 \\ Received: May 6, 2017, 2017 \\ Accepted: May 26, 2017 \\ Published: November 1, 2017 \\ Copyright: (๑) 2017 Clarence S Yah. This \\ is an open access article distributed under \\ the terms of the Creative Commons \\ Attribution License, which permits \\ unrestricted use, distribution, and \\ reproduction in any medium, provided the \\ original author and source are credited. \\ Funding: Nil \\ Competing Interests: The authors \\ declare that this manuscript was approved \\ by all authors in its formand that no \\ competing interest exists. \\ Affiliation and Correspondence: \\ ${ }^{1}$ Implementation Science Unit, Wits \\ Reproductive Health and HIV Institute \\ (Wits RHI), Faculty of Health \\ Sciences, University of the \\ Witwatersrand, Johannesburg, South \\ Africa \\ Email: cyahsuh@gmail.com
}

\section{ABSTRACT}

BACKGROUND: Despite the shift in antiretroviral therapy (ARVs) eligibility cascade from $C D 4 \leq 200$ to $C D 4 \leq 350$ to $C D 4 \leq 500$ $\mathrm{mm}^{3}$, HIV related morbidity and mortality continue to escalate annually, as do HIV infections. The new paradigm of treatment for all HIV positives individual irrespective of CD4 count may significantly reduce HIV and related illnesses. The author assumes that all HIV infected partners should be eligible for HIV treatment and care, irrespective of CD4 count. A second assumption is that high risk HIV negative partners have free access to continuum of HIV pre-exposure prophylaxis (PrEP), post exposure prophylaxis $(P E P)$ and other prevention packages.

METHODS: A literature review search was used to extract evidence-based ARVs-HIV treatment and prevention interventions among HIV positives and high risk partners respectively. Only articles published in English and indexed in journal nuclei were used for the study. The information was used to nurture understanding of HIV treatment and prevention approaches as well as HIV incidence multiplier effect among HIV serodiscordant partners. The imputed HIV incident reference was assumed at 1.2 per 100 person-years (2). This was based on the imputation that retention in care, adherence and other predetermined factors are functions of an effective health care delivery system.

RESULT: The model showed a reduced HIV transmission from 1.2 per 100 person-years to 1.032 per 100 person-years in 6 months. The average threshold period of HIV suppressed partners on ARVs to an undetectable level. The combined multiplier protective-effect probability of transmitting HIV from HIV positive partners on ARVs-suppressed viremic load to HIV negative partners on PrEP/PEP-prevention was detected at $86 \%$

CONCLUSION: The model showed a significant reduction in HIV incidence. Placing serodiscordant sexual partners in HIV treatment and prevention plays a significant role in reducing and controlling HIV infection. Therefore, the policy of enrolling all HIV positives irrespective of CD4 count on ARVs and high risk partners on prevention if adopted and sustained may underpin reduction and control of HIV genotype and HIV related morbidity, mortality and opportunistic infections.

KEYWORDS: HIV, test and treat, linkage to care, prevention, incidence 


\section{INTRODUCTION}

HIV infection is the most widely sensitized, researched, implemented and costly pandemic intervention program the world is still struggling to contain. According to estimates by UNAIDS, 31.1 billion US Dollars is projected to be spent by 2020 and US\$29.3 billion by 2030 to contain HIV and related morbidities and mortalities (6). The fatality rate is over 30 million people (7), and currently over 36 million people are living with the virus with an estimated 17 million accessing ARVs globally(8).

Since the introduction of ARVs eligibility from CD $4 \leq 200$ to $\mathrm{CD} 4 \leq 350$ and to $\mathrm{CD} 4 \leq 500$ $\mathrm{mm}^{3}$ cascade, HIV new infections and related disease burden continued to escalate annually. With the introduction of combination prevention packages of HIV testing services (HTS) and ARVs treatment, coupled with sustained partnership with local and international stakeholders in containing the epidemic, new HIV infections have dropped by $35 \%$ since 2000 , including HIV related mortality by $42 \%$ (6). Evidence-based researches reveal continuum of HTS, HIV treatment coverage and ARVs prevention to play a significant role in reducing HIV-viremic transmission and acquisition (1-3).

Furthermore, active social mobilization engagement and empowerment of local communities are additional behavioural change communications that have enhanced acceptability and uptake of HIV prevention and health seeking behaviour (9). Others include the utilization of sexual reproductive health (SRH) services and right to venerable population such as adolescents (10-12, 35). According to Williams et al (7), social, behavioural, biomedical and community level testing have enhanced and improved acceptability and uptake of health services. These evidence-based interventions have played a significant role in reducing HIV incidences in subSaharan Africa.

By engaging all partners in the algorithm of HTS and treatment cascade irrespective of CD4 count, retention in care, adherence and vigorous, active prevention strategies are essential innovative strategies in achieving the 90-90-90 HIV objectives especially in high risk vulnerable settings $(13,14)$ including key populations (12) and adolescents-girls $(11,12)$. In addition, the current concept of enrolling all those who test positive for HIV on treatment and linkage to care irrespective of CD4 count and active access to PrEP, PEP, condoms, lubes and treatment of other sexually transmitted infections (STIs) have emerged as a new expansion of care and prevention. The implementation may account for HIV genotype containment globally. The author therefore assumed that all HIV infected partners should be eligible for HIV treatment and care, irrespective of CD4 count. A second assumption is that high risk HIV negative partners have free access to continuum of PrEP, PEP and other prevention packages because they are in HIV serodiscordant relationship. The proposed model describes the HIV incidences with the assumption that all HIV infected partners are eligible to ARVs treatment and linkage to care irrespective of CD4 count. The trajectory is based on the assumption that HTS and ARVs quality in routine health service deliveries are prioritized in these setting.

\section{METHODS}

A literature review search design was used to extract evidence-based high quality ARVs-HIV treatment and prevention interventions among HIV positives and high risk partners. The articles included in the study were those published in English and provided clear description of HIV rate due to ARVs treatment (96\%) (1-3) and prevention (PrEP and PEP) interventions (90\%) (4).

Keywords such as "antiretroviral treatment and viral load reduction/suppression", "HIV treatment and prevention among serodiscordant partners" and "PrEP, PEP amongst HIV negative partners" were used for the search. Serodiscordant relationship (partner) was defined as one being partner HIV positive and the other being HIV negative and likely to acquire HIV from the HIV positive partner. The following indexing journal nuclei were used for the search:

DOI: http://dx.doi.org/10.4314/ejhs.v27i6.7 
Cochrane Library, PubMed, MeSH PsycInfo, Scopus and Google Scholar, Embase and Global Health. The synthesised evidence-base interventions were used to nurture understanding of HIV treatment and prevention approaches. This was based on ARVs-viral load suppression among HIV positives and HIV prevention amongst sexually active high risk partners on either PrEP or PEP. The imputed HIV incident in the study was assumed at 1.2 per 100 person-years (2).

This assumption was based on the fact that retention in care, adherence and other predetermined factors are functions of an effective health care delivery system. The predetermined factors include arrays of behavioural, socioeconomic and structural factors related to HIV acquisition and transmission which were highlighted as constant variables in the model (28). Inclusive were factors such as mobility and migration, cultural practices (circumcisions) and educational status, early sexual debut and sexual violence (15). Other constants included were alcohol and drug use, condom use $(13,14)$, and mother to child transmission of HIV (MTCT) during pregnancy and post natal care (16).

The model HIV treatment and preventions approaches: The study estimated HIV transmission in all suppressed viremic partners on ARVs to HIV negative partners at approximately 4\% (1-3) and HIV acquisition from HIV positive partners to HIV negative partners on PrEP and PEP at approximately $10 \%(4,7)$. This model therefore accounted for 2 cascades: treatment cascade (HIV positive-ARV treatment) and prevention cascade (HIV Negative-PreP and PEP), while retention in care, adherence, STIs treatment and other variables are held constant and imputed as a function of an effective health care delivery quality. Entry to the model were HIV positive individuals on ARVs care and PMTCT programme. Linkage to care cascade are all who test positive for HIV, irrespective of CD4 count and whether they are on HIV treatment or not. These were called HIV transmission partners, while those in the prevention arm are high risk/exposed HIV negative partners' accessing PrEP or PEP. These were termed HIV acquisition partners.
Estimating HIV incident using $100 \%$ test and treat, linkage to care irrespective of CD4 count and prevention strategies: Antiretrovirals (ARVs) have been tested, proved and defined as a critical pointer in controlling HIV replication, reduction in viremic load, transmission and maintaining of health quality (1-3). There are several predictor factors associated with HIV treatment and ARVs administration including ARVs-partner specific properties, the virus characteristics, patient-specific variables, environmental variables as well as patient provider relationships (19). In the model, these variables were imputed as constant although they do play a significant role in HIV transmission and acquisition.

Note that the prime entries into the model are: sustained ARVs or PMTCT and linkage to care all HIV positive partners irrespective of CD4 count and free access to HIV preventions (PrEP/PEP) sexually high risk/exposed HIV negative partners.

Using the basic reproduction number (R0), which is the expected number of secondary new HIV infections from an infected partner to a completely HIV negative population as defined in equation 1. The model uses this to highlight the transmission of HIV from an infected partner to susceptible partners.

$\mathrm{R} 0=\mathrm{T} . \mathrm{C} . \mathrm{D} \ldots \ldots \ldots \ldots \ldots \ldots$..............

Where $\mathrm{T}=$ Transmissibility probability of infection given contact between a susceptible and HIV infected partner. $\mathrm{C}=$ Average rate of contact between susceptible and HIV infected partner, $\mathrm{D}=$ Duration of infectiousness.

The model considers retention in care, adherence and other variables as constants. This implies that infected HIV partners who are fully on ARVs or PMTCT compliance have low undetectable viremic load and a $96 \%$ chance of not transmitting HIV to HIV negative partners. While those on HIV preventions such as PrEP and PEP have a $90 \%$ chance of not acquiring HIV from HIV positive partners during sexual intercourse. Therefore, if R0 can be sustained and substantially reduce to less than 1 due to viral load suppression by ARVs. This may significantly reduce new HIV infections.

DOI: http://dx.doi.org/10.4314/ejhs.v27i6.7 
Based on equation 1 and other sources, we assumed the probability of all virally suppressed HIV positive partners on ARVs, transmission of HIV to susceptible partners at $96 \%(\mathrm{p}=0.96)(1-$ 3). Equation 2

Also the probability of sexually active HIV negative partner on PrEP or PEP acquiring HIV from infected partner is estimated at $90 \%(\mathrm{q}=0.9)$ $(4,5) \ldots \ldots \ldots \ldots . .$. Equation 3.

Therefore, the multiplication effect of transmission and acquiring probability from equations $2 \& 3=$ p x q $=(0.96 \times 0.9)=0.86$ $(86 \%) \ldots \ldots \ldots \ldots . . .$. Equation 4.

This implies that the combined probability effect of transmitting HIV from HIV positive partners on ARVs-suppressed viremic load to HIV negative partners and HIV negative partners on PrEP/PEP-prevention is approximately 0.86 equating to $86 \%(4,7)$. In addition, according to a study by Cohen et al (2), the incident rate among serodiscordant stable HIV relationship where one partner is fully on ARVs is estimated at 1.2 per 100 person-years. Mathematically, the combined probability of transmitting and acquiring of HIV at incident rate of 1.2 per 100 person-years will be estimated at $1.032(0.86 \times 1.2)$ per 100 personyears provided other factors are held constant including ARVs-resistant HIV strains.

Studies show that retention and adherence in ARVs for 6 months account for viremic suppression to undetectable level in plasma (20). This implies that retention in care and adherence in ARVs treatment and prevention for 6 months can halt HIV transmission and acquisition among serodiscordant partners during sexual intercourse by $86 \%$. This can significantly reduce HIV incidences including morbidity and mortality of HIV related infections. Figure 1 below depicts a simple algorithm model of HIV transmission and acquisition in a disproportionally high risk and exposed serodiscordant partners.

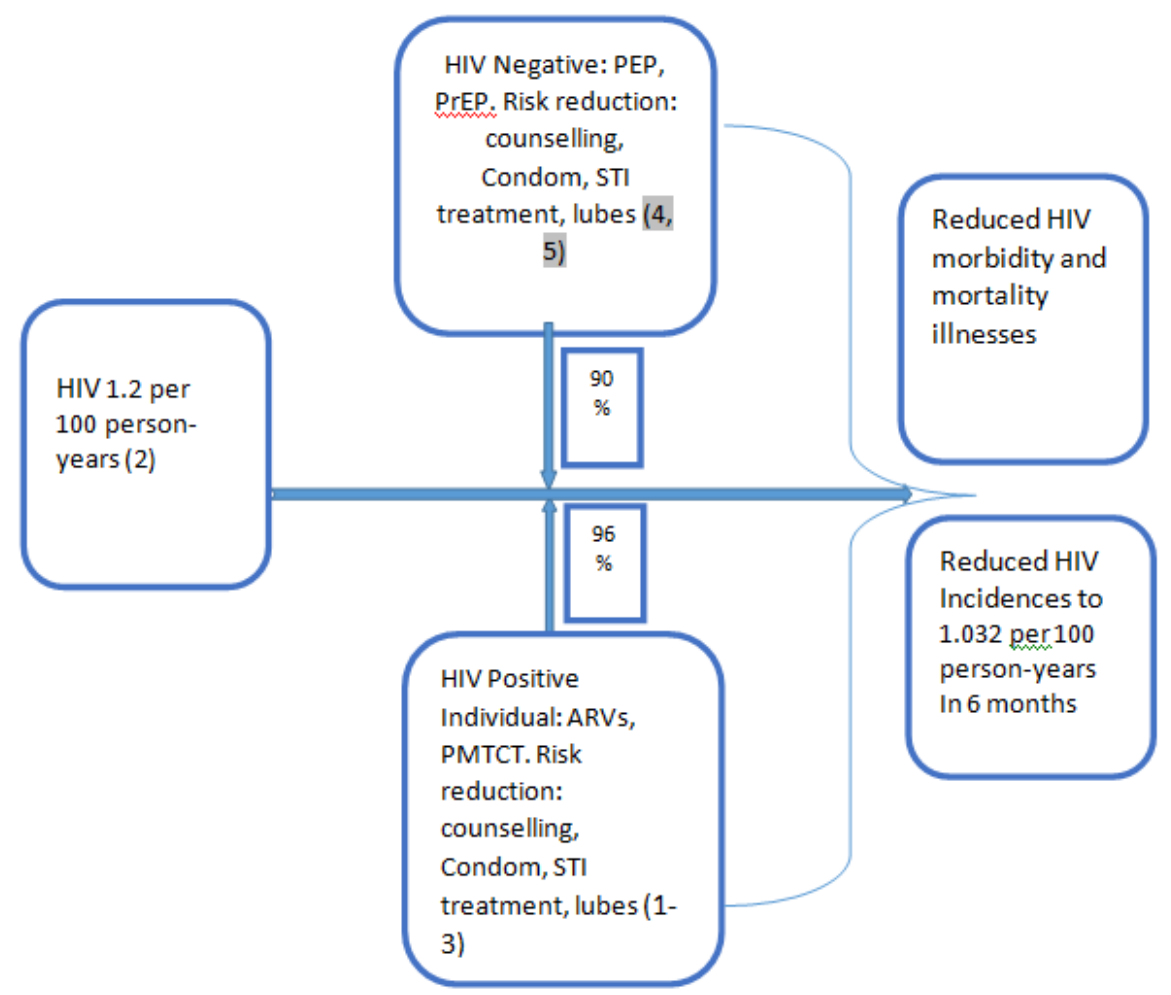

Figure 1: HIV ART, prevention and incidence cascade

DOI: http://dx.doi.org/10.4314/ejhs.v27i6.7 


\section{RESULTS}

According to findings by Cohen et al (2), HIV transmission rate among serodiscordant partners eligible to CD4 count and ARVs-treatment is estimated at 1.2 per 100 person-years. The average threshold period of HIV suppressed partners on ARVs to an undetectable level. While the adoption of test and treat model among serodiscordant partners irrespective of CD4 count and ARVstreatment reduces the HIV incidence from 1.2 per 100 person-years to 1.032 per 100 person-years. The combined multiplier protective-effect probability of transmitting HIV from HIV positive partners on ARVs-suppressed viremic load to HIV negative partners on PrEP/PEP-prevention was detected at $86 \%$ as shown in Figure 1.

\section{DISCUSSION}

Continuum of HIV counselling and testing (HCT) and couple voluntary counselling and testing (CVCT): Without HCT/CVCT, the probability of estimating the burden of the disease and accounting for the 90-90-90 cascade will be a failure. HIV counselling and testing is the prime gateway and entry point into HIV treatment, care and prevention (17). Although operational and continuous imperative logistics including shortages of HCT consumables and health care providers, skilled-competent counsellors and community health workers may be hindering sustain acceptability, uptake and adoption of HCT/CVCT. The 90-90-90 sustained HCT/CVCT acceptability, uptake and adoption require a robust-decentralized heath service delivery system. This includes capacity development, support structures, community decentralization of HCT and the integration of community HCT services into the national delivery HCT testing and treatment grid. The downstream strengthening of HCT/CVCT services plays an essential role in bridging positive changes and sexual behaviour of other STIs, HIV treatment and care, retention and adherence. Other hindering variables include partner disclosure, stigma and discrimination, positive living and positive prevention, psychosocial support, mental health and HIV related opportunistic infections treatment as well as tuberculosis (TB). The current HTS has incorporated point-of care (POC) testing such as POC-CD4 and POC-creatinine and on-site ARVs initiation and linkage to care and services (29). This is accompanied by direct communication and collaboration with local clinics where partners can receive further referral and access to drugs (18). Continuum of HIV treatment and prevention cascade: To fulfil the 90-90-90 HIV test, treat, and viral load suppression approach irrespective of CD4 count especially in sub-Saharan Africa, countries such as Namibia and South Africa are already implementing the program to all HIV positives and serodiscordant partners. The recommendations are based on real time evidencebase findings that people who start ARV treatment earlier get better health outcomes and are likely to reduce HIV transmission in serodicordant relationship (1-3,33). HIV-positive partners on ARVs with suppressed viral load to undetectable level have more than $96 \%$ chances of not transmitting the virus to their HIV negative sexual partners $(1-3,33)$ while those on PrEP and PEP have more than $90 \%$ chances of not acquiring HIV from HIV positive partners (4). The mathematical modelling from studies showed a combined treatment and prevention effect among serodiscordant partners (ARVs-treatment and PrEP/PEP-prevention) at $86 \%$ chances of not transmitting and acquiring HIV vice versa. Placing serodiscordant sexual partners in HIV treatment and prevention can play a significant role in reducing and controlling HIV.

Use of innovative drug dispensing, pharmacy, community, national-use of $M$-health and $E$ health systems: Scaling up access from the current 16 million ARVs treatment and care to over 36-million living with HIV globally, require a sustained adoption of test and treat ARVs supply-chain management policy which is a function of a successful health care system. This include innovative strategies that maximise the availability and dispensing of drugs. Information and communication technology (ICT) including digital electronic health (E-Health), messaging health (M-Health) and social media at the centre of effective health systems support (33). This provide and support partners, communities, 
national, regional and international on effective public health services (33). The use of ICT has improved partner perception of HIV knowledge in many settings $(30,31)$. The dynamism of drug automated pharmacy dispensing machines, similar working mechanisms as banks automated teller machines (ATM) are key components that can reduce HIV structural factors of stigma and discrimination while increasing drug uptake, retention and adherence in HIV care as well as access to HIV drugs which in turn, improve health outcomes(21). The use of drug automated pharmacy dispensing machines provide 24 hour hour access to ARVs and other medications (21). Evidence-based studies have highlighted the importance of E-Health, M-Health in reducing waiting time at health care centres and hospital settings (33). The use of M-Health reminds partner of medication uptake which has been highly associated with sustained viral suppression and better health outcomes (32). The use of innovative ARVs automated pharmacy dispensing machines and holistic integrated pharmacy and community drug dispensing system, including national-use of ATM medication dispensing could enhance innovative strategies in retaining and adhering partner in treatment and care. This can significantly reduce waiting time in clinical settings. However, the lack of ICT operations, infrastructures and investment in developing countries are limiting factors hindering integrated digital health systems (36).

Fostering combination prevention approach: Despite the significant role of ARVs, a combination approached of behavioural, biological and community level engagement are additional strategies in containing HIV transmission and acquisition $(4,9,14,32)$, each contributing a synergic effect. Figure 2 depicts a synergic illustration approach of HIV transmission and acquisition containment including condom use (22), microbicides (23), circumcision(24), PMTCT(25), STIs treatment $(4,9,14)$, behavioural change and community engagement $(9,33)$.

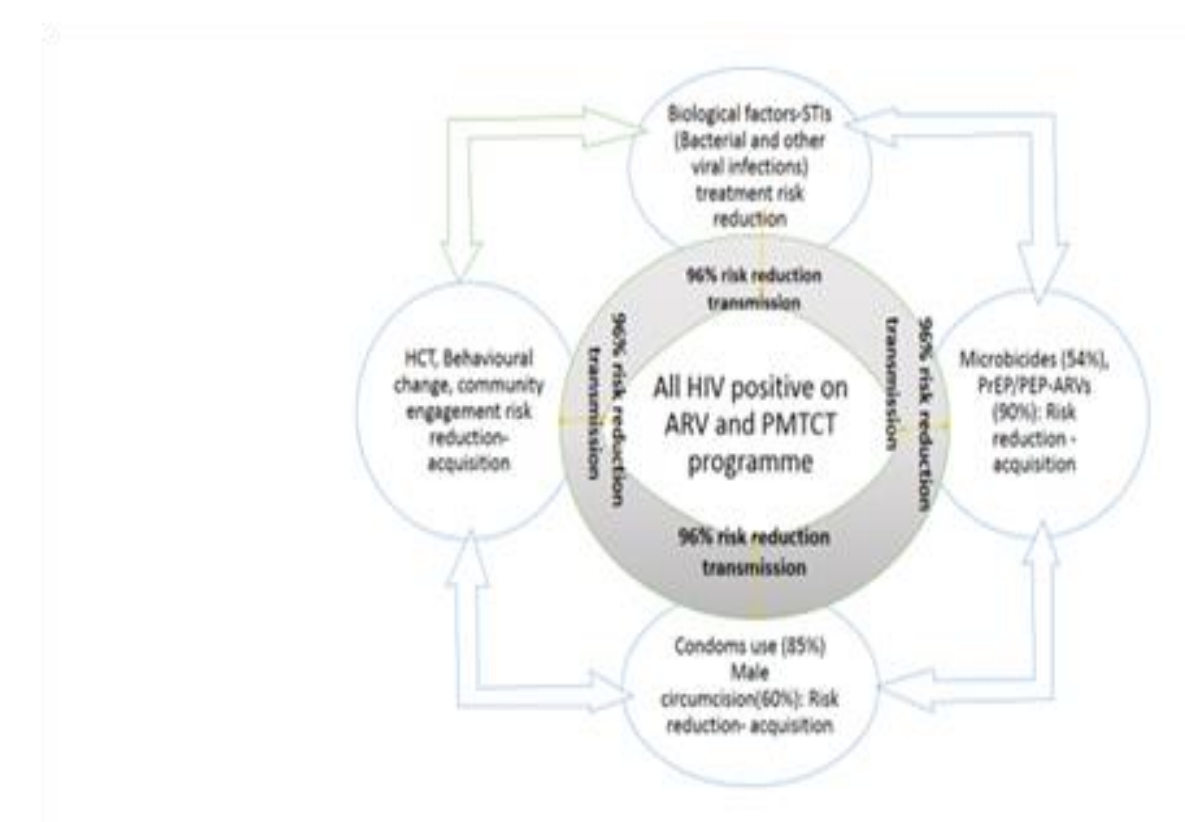

Fig 2: Combination approach of HIV transmission and treatment cascade framework

DOI: http://dx.doi.org/10.4314/ejhs.v27i6.7 
HIV treatment/prevention, retention in care and adherence are key components of continual suppression of HIV (26). The enrolment and access to ARVs irrespective of CD4 count and access to HIV prevention by HIV negative naïve population will reduce HIV transmission and acquisition. The model showed a significant reduction in HIV incidence. Placing serodiscordant sexual partners in HIV treatment and prevention can play a significant role in reducing and controlling HIV. Therefore, the policy of enrolling all HIV positives irrespective of $\mathrm{CD} 4$ count on ARVs and high risk partners on prevention if adopted and sustained may underpin reduction and control of HIV genotype. This will significantly reduce HIV related morbidity, mortality and opportunistic infections. In addition, the policy will redefine the future of HIV transmission and acquisition as well as access to health care services. The use of ICT in changing healthcare practice, outcomes and perceptions will improve health care accessibility. However, in most African settings, ICT operational and resources are still at infancy.

Furthermore, ARVs contextual and multifactorial components of HIV risk reduction are proactively defined at partner level, community, national and public health levels (27). Although health system factors including long waiting times, drugs supplies, infection control, staff attitude and other cross-cutting structural elements may affect the acceptability and sustained uptake of HCT, ARVs care, viral load suppression and HIV prevention strategies (37). Programmes with better understanding of socio-cultural, economic, demographic and structural barriers to HIV treatment may have potential impact on HIV incidence.

\section{ACKNOWLEDGMENTS}

I am grateful to the mathematical simulation discussions and inputs from Prof Geoffrey Simate School of Chemical Engineering, University of the Witwatersrand as well as the valuable input and suggestions from Dr. Eustasius Musengue of Wits School of Public Health.

DOI: http://dx.doi.org/10.4314/ejhs.v27i6.7 


\section{REFERENCES}

1. CDC. Achievements in public health: reduction in perinatal transmission of HIV infection-United States. NMMWR 2006; 55: 592-597.

2. Cohen MS, Chen YQ, McCauley M, Gamble T, Hosseinipour MC, Kumarasamy N, Hakim JG, Kumwenda J, et al. 'Prevention of HIV-1 Infection with Early Antiretroviral Therapy. The New England Journal of Medicine 2011; 365(5): 493-505.

3. Quinn TC, Wawer MJ, Sewankambo N, Serwadda D, Li C, Wabwire-Mangen F, Meehan MO, Lutalo T, Gray RH. Rakai Project Study Group. Viral load and heterosexual transmission of human immunodeficiency virus type 1 . Rakai Project Study Group. Engl J Med 2000; 42(13): 921929.

4. Baeten JM, Donnell D, Ndase P, Mugo NR, Campbell JD, Wangisi J, Tappero JW, Bukusi EA, et al. Partners PrEP Study Team. Antiretroviral prophylaxis for HIV prevention in heterosexual men and women. $N$ Engl $J$ Med 2012; 367(5): 399-410.

5. Donnell D, Baeten JM, Hendrin C. et al. Tenofovir drug levels indicated PrEP use is strongly correlated with HIV-1 protective effects. Paper presented at the 19th Conference on Retroviruses and Opportunistic infections (CRO). March 5-8, 2012. Seatle, WA. 12. , 2012.

6. UNAIDS. GLOBAL STATISTICS. Fact sheet 2015, 2015.

7. Williams BG, Lima V, Gouws E. Modelling the impact of antiretroviral therapy on the epidemic of HIV. Curr HIV Res. 2011; 9(6): 367-382.

8. UNAIDS. Global AIDs update. 2016 report from UNAIDS. 2016.

9. Molyneux S, Sariola S, Allman D, Dijkstra M, Gichuru E, Susan Graham, Kamuya D, Gakii G, Kayemba B, Kombo B, Maleche A, Mbwambo J, Marsh V, Micheni M, Mumba N, Parker M, Shio J, Yah C, van der Elst E, Sanders E. Public/community engagement in health research with men who have sex with men in sub-Saharan Africa: challenges and opportunities. Health Research Policy and Systems 2016; 14(1): 14.

10. Ngomi KB. Utilisation of Sexual and Reproductive Health Services by Secondary School Adolescents In Mochudi. MASTER OF ARTS Thesis. Submitted in the fulfilment of the requirements for the degree of master of Arts. University of South Africa, South Africa. 2008.

11. Suthar AB, Ford N, Bachanas PJ, Wong VJ, Rajan JS, et al. Towards Universal Voluntary HIV Testing and Counselling: A Systematic Review and Meta-analysis of CommunityBased Approaches. PLoS Med 2013; 10(8): 10001496.

12. UNAIDS. 90-90-90 An ambitious treatment target to help end the AIDS epidemic. Joint United Nations Programme on HIV/AIDS (UNAIDS). UNAIDS / JC2684, 2014.

13. Stahlman S, Johnston LG, Yah C, Ketende S, Maziya S, Trapence G, Jumbe V, Sithole B, Mothopeng T,Mnisi Z, Baral S. Respondentdriven sampling as a recruitment method for men who have sex with men in southern subSaharan Africa: a cross-sectional analysis by wave. Sex Transm Infect 2016; 92(4):292-8.

14. McNaghten A, Kearns R, Siegler AJ, Phaswana-Mafuya N, Bekker LG, Stephenson $\mathrm{R}$, Baral SD, Brookmeyer R, Yah CS, Lambert AJ, Brown B, Rosenberg E, Blalock Tharp M, de Voux A, Beyrer C, Sullivan PS. Sibanye Methods for Prevention Packages Program Project Protocol: Pilot Study of HIV Prevention Interventions for Men Who Have Sex With Men in South Africa. JMIR Res Protoc 2014; 3(4): e55.

15. Haberland N. The case for addressing gender and power in sexuality and HIV education: A comprehensive review of evaluation studies International Perspectives on Sexual and Reproductive Health 2015; 41(1).

16. Darbes LA1, van Rooyen H, Hosegood V, Ngubane T, Johnson MO, Fritz K, McGrath N. Uthando Lwethu ('our love'): a protocol for a partners -based intervention to increase 
testing for HIV: a randomized controlled trial in rural KwaZulu-Natal, South Africa. Trials 2104; 20(15): 64.

17. Mabuto T, Latka MH, Kuwane B, Churchyard GJ, Charalambous S, Hoffmann CJ. Four Models of HIV Counseling and Testing: Utilization and Test Results in South Africa. PLoS ONE 2014; 9(7): e102267.

18. Bigdeli M, Tomson G, Laing R, Ghaffar A, Dujardin B, Van Damme W. Access to medicines from a health system perspective. Health Policy Plan 2012; 28(7): 692-704.

19. Machtinger EL, Bangsberg DB. Adherence to HIV Antiretroviral Therapy. http://hivinsite.ucsf.edu/InSite?page=kb-0302-09 2006.

20. Smith CJ, Staszewski S,Sabin CA, Nelson M, Dauer B, Gute P, Johnson MA. Phillips AN, Gazzard B. Use of viral load measured after 4 weeks of highly active antiretroviral therapy to predict virologic outcome at 24 weeks for HIV-1-positive individuals. J Acquir Immune Defic Syndr 2004; 37: 1155-1159.

21. Gheihman G. Ontario's Remote Drug Dispensing Policy as an Innovation to Improve Prescription Medication Access in Remote, Rural, and Northern Communities. Health Council of Canada Health Innovation Challenge 2011/2012. 2012.

22. Fayaz AM, Ao Z, Girilal M, Chen L, Xiao X, Kalaichelvan $\mathrm{PT}$, Yao $\mathrm{X}$. Inactivation of microbial infectiousness by silver nanoparticles-coated condom: a new approach to inhibit HIV- and HSV-transmitted infection. International Journal of Nanomedicine 2012; 7: 5007-5018.

23. Abdool Karim QA, Karim SSA, Frohlich JA, et al. Effectiveness and safety of tenovofir Gel and antiretroviral microbicide for the prevention in men who have sex with men. $N$ Engl J Med 2010; 3:2587-2599.

24. Auvert B, Taljaard D, Lagarde E, SobngwiTambekon J, Sitta R, Puren A. Randomized control intervention trial of male circumcision for reduction of HIV infection risk: The ANRS 1265 trial. Plos Med 2005; E298.

25. European-collaborative-study. Mother-tochild-transmission of HIV infection in the era of highly active antiretroviral therapy. Clin Infect Dis 2005; 40: 458-465.

26. Mountain E, Mishra S, Vickerman P, Pickles M, Gilks C, Boily MC. Antiretroviral Therapy Uptake, Attrition, Adherence and Outcomes among HIV-Infected Female Sex Workers: A Systematic Review and Meta-Analysis. PLoS ONE 2014; 9(9): e105645.

27. Hodgson I, Plummer ML, Konopka SN, Colvin CJ, Jonas E, Albertini J, et al. A Systematic Review of Individual and Contextual Factors Affecting ART Initiation, Adherence, and Retention for HIV-Infected Pregnant and Postpartum Women. PLoS ONE 2014; 9(11): e111421.

28. Munro S, Lewin S, Swart T, Volmink J. A review of health behaviour theories: how useful are these for developing interventions to promote long-term medication adherence for TB and HIV/AIDS? BMC Public Health BMC series. 2007; 7:104.

29. Vojnov L, Markby J, Boeke C, Harris L, Ford $\mathrm{N}$, Peter T. POC CD4 Testing Improves Linkage to HIV Care and Timeliness of ART Initiation in a Public Health Approach: A Systematic Review and Meta-Analysis. PLoS One 2016; 11(5): 0155256.

30. Catalani C, Philbrick W, Fraser H, Mechael P, Israelski DM. mHealth for HIV Treatment \& Prevention: A Systematic Review of the Literature. Open AIDS Journal 2013; 7:17-41.

31. Berrouiguet $S$, Baca-García $E$, Brandt $S$, Walter M, Courtet P. Fundamentals for Future Mobile-Health (mHealth): A Systematic Review of Mobile Phone and Web-Based Text Messaging in Mental Health. J Med Internet Res 2016; 18(6):e135

32. Pop-Eleches C, Thirumurthy H, Habyarimana JP, Zivin JG, Goldstein MP, de Walque D, MacKeen L, Haberer J, Kimaiyo S, Sidle J, Ngare D, Bangsberg DR. Mobile phone technologies improve adherence to antiretroviral treatment in a resource-limited setting: a randomized controlled trial of text message reminders. AIDS 2011; 25(6):825-34.

33. Yah CS, Tambo E, Khayeka-Wandabwa C, Ngogang JY. Impact of telemonitoring approaches on integrated HIV and TB 
diagnosis and treatment interventions in subSaharan Africa: a scoping review. Health Promotion Perspectives 2017; 7(2):60-65.

34. Yang RR, Gui X, Xiong Y, Gao SC, Yan YJ. Five-year follow-up observation of HIV prevalence in serodiscordant partners. International Journal of Infectious Diseases 2015; 33:179-84.

35. 35. Stahlman S, Johnston LG, Yah C, Ketende S, Maziya S, Trapence G, Jumbe V, Sithole B, Mothopeng T, Mnisi Z, Baral S. Respondent-driven sampling as a recruitment method for men who have sex with men in southern sub-Saharan Africa: a cross-sectional analysis by wave. Sexually Transmitted Infections 2016; 92(4):292-8.
36. Tambo E, Madjou G, Mbous Y, Olalubi OA, Yah C, Adedeji AA, Ngogang JY. Digital Health Implications In Health Systems In Africa. European Journal of Pharmaceutical and Medical Research, 2016;3(1), 91-93.

37. Schwartz S, Lambert A, Phaswana-Mafuya N, Kose Z, Mcingana M, Holland C, Ketende S, Yah C, Sweitzer S, Hausler H, Baral S. Engagement in the HIV care cascade and barriers to antiretroviral therapy uptake among female sex workers in Port Elizabeth, South Africa: findings from a respondent-driven sampling study. Sex Transm Infect. 2017;93 (4): 290-296. 